\title{
THE PATHOLOGY OF THE PAINFUL HEEL
}

\author{
H. C. BURRY, M.R.C.P., M.R.A.C.P., D.Phys.Med.
}

Guy's Hospital, London S.E.1.

The mythological derivation of the term 'Achilles Heel' is such as to denote a localized point of vulnerability. The tendon which bears the name of Achilles has maintained that reputation, and is the site of perhaps the most persistent and annoying lesion known to modern athletes. Successful management of the painful lesions of this area demands accurate diagnosis and an awareness of the anatomical and pathological factors involved.

\section{Anatomical Considerations.}

The tendo-achilles is composed of the rather flat soleus tendon with the gastrocnemius tendon superficial to it, and partly enveloped on either side by it - that is the soleus tendon is U-shaped in cross section. The associated plantaris tendon is small in size and importance. The tendo-achilles is inserted into the lower posterior surface of the calcaneum (os calcis), being separated from the upper posterior surface by a bursa. It is separated from the deep flexors by the deep transverse fascia and from the skin by loose areolar tissue. During flexion and extension skin and tendon tend to move together so that there is little friction. Friction effects are more apt to occur on the deep surface, either between tendo-achilles and deep fascia, or in the bursa between tendon and os calcis. An adventitious bursa may also be present between the superficial surface of the tendon and the overlying skin. It is probably of relevance in the genesis of lesions of the tendon, that the two components (i.e. gastrocnemius and soleus) have vastly different sites of origin, and therefore function. The soleus arises from the posterior surfaces of tibia and fibula, but the gastrocnemius arises in two heads from the medial and lateral condyles of the femur. Thus gastrocnemius is required to function over two joints, while soleus is concerned only with the action of the ankle joint. This being so, it is clear that the two components of the tendon in their upper parts must move independently of each other in some movements.

The Achilles tendon is easily the strongest in the body, being capable of withstanding forces of five to ten kilograms per square millimetre, (Elliott, 1965) that is roughly one thousand pounds in an average adult. It is composed of compact bundles of collagenous fibres, and is surrounded by a sheath, the paratenon. The blood supply is tenuous, reaching the tendon from its sheath, and is almost certainly considerably affected by changes of pressure within the sheath. Although the metabolic activity of tendon is low, there is a continuous process of turnover of collagen, and chronic ischaemia leads to atrophy, while acute ischaemia is followed by mucoid degeneration. In addition, and perhaps related to vascular changes, tendon in common with other connective tissue atrophies with increasing age.

It is as well that the tendon is strong, since it is subject to great stresses. If the foot is regarded as a lever with the ankle joint as the fulcrum, it is readily appreciated that the triceps surae operates the short arm of a first order lever, and has a considerable mechanical disadvantage, approximately three to one. In the moments of thrusting off from the toes or alighting triceps is required to produce plantar flexion or control dorsiflexion. A two-hundred pound man may thus require a force of six hundred pounds to lift him from the ground.

Pathological Classification of Lesions.

\section{Lesions of the tendon itself.}

(a)Complete rupture:

1. Following previous mucoid degeneration.

2. Without antecedent pathology.

(b)Minor lesions - rupture of a small number of fibres, occurring:

1. At insertion

2. Within the tendon

3. At the musculo-tendinous junction.

\section{Peritendinous:}

Inflammatory conditions of:

(a) The calcaneal bursa.

(b)The sub-cutaneous bursa.

(c)Peritendinous connective tissue.

\section{Rupture of the tendon.}

In view of the apparent safety margin between intrinsic strength and maximal strains it is perhaps surprising that rupture ever occurs in the healthy subject.

In one series of 33 cases of complete rupture, ranging in age from $6-70$, the average was 40. (Goldman, Linscheid and Bickel, 1969) Two-thirds occurred during participation in some form of sport. 
It was found possible to rupture the Achilles tendons of fit but old wild rats, but not those of unfit laboratory animals. Clearly other factors may have been operating in this case, but the implication is that a strong muscle is required before rupture is likely to occur. Furthermore histological examination did not show any evidence of pathological changes within the tendons. However, the preponderance of ruptures in older age groups suggests that the known atrophy of connective tissue with increasing age is an important factor, and in many cases areas of degeneration are apparent. (Ljungquist, 1968); (Arner, Lindholm and Orell, 1958) The relationship of the latter to previous local corticosteroid therapy is not clear, but there is some evidence that injections of cortico-steroid within the paratenon may not only weaken the tendon by suppressing collagen synthesis, but may also, by increasing the pressure within the paratenon, embarrass the blood supply and lead to ischaemic necrosis. Ljungquist's findings however, do not support this view.

\section{Minor Lesions of the Tendon.}

Perhaps the most important lesion of the tendon in terms of loss of function and long term disability is the minor lesion. Clinically this causes persistent pain which tends to settle after reduction of the training schedule, but recurs and finally becomes unbearable with stepping up of the load. These lesions are characterised by the presence of a small exquisitely tender nodule in the tendon, sometimes accompanied by swelling of the peritendinous tissues. When the tendon is opened, a careful search will expose the presence of a small area of degeneration in the tendon, Histologically the collaginous fibres have lost their characteristic wavy appearance and the outline of the fibres is blurred. The nuclei of the fibroblasts are pyknotic. Associated with this area of degeneration are small groups of vascular channels, mainly thin-walled sinuses. A few chronic inflammatory cells are also seen. Fig. 1.2. 3.

Lesions of this nature are most common at a point approximately one and a half inches above the insertion of the tendon, but they may occur elsewhere and are not uncommon at the point of insertion, being then analagous to the common lateral epicondylitis (Tennis Elbow).

Lesions at the musculo-tendinous junction tend to be more acute in onset, and are less likely to become chronic, probably because of the superior blood supply to this region.

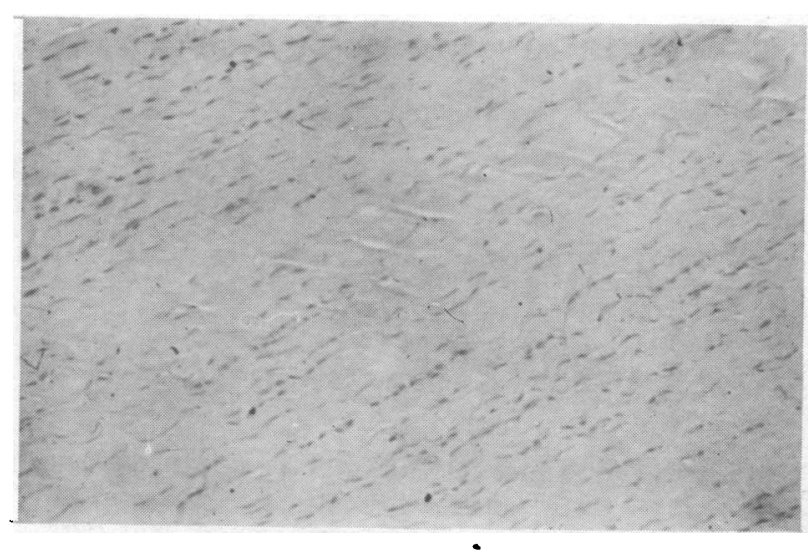

Fig. 1 Normal Tendon. Note characteristic wavy outline of collagen fibres. H.E. $x 31.3$

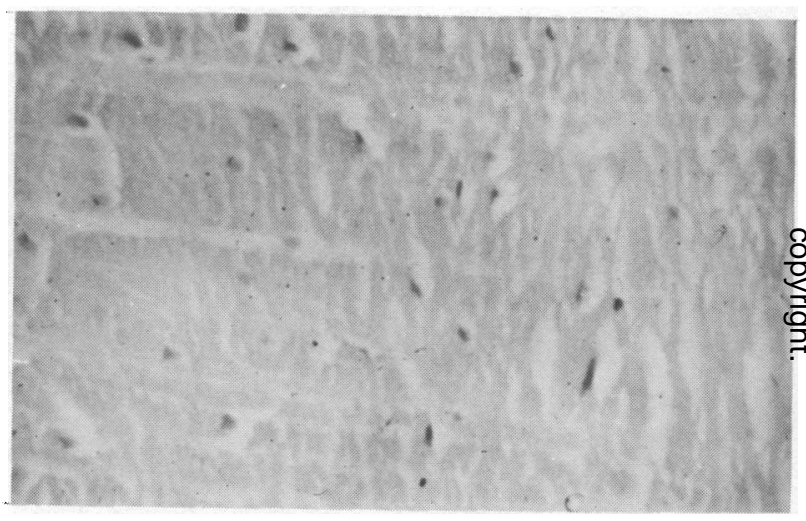

Fig. 2 Necrotic collagen. Fibres and pyknotic fibrolast nuclei. H.E. $\times 125.2$

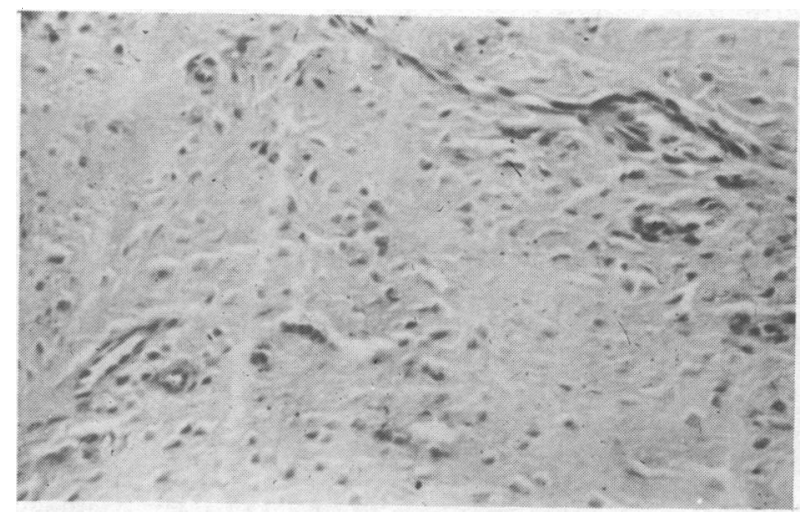

Fig. 3 Area of granulation in tendon. H.E. $\times 77.8$ 


\section{Peritendinous Lesions.}

The most common lesion causing painful heel is a diffuse inflammation of the peritendinous areolar tissue. It is likely that this is a direct friction effect, arising from overuse or strain, and probably has its origin at the posterior surface of the tendon where it lies against the transverse fascia, since careful examination usually localises the point of maximum tenderness in this region. The associated swelling tends to be diffuse, filling up the normal groove between the posterior surface of the tibia on the medial side, and the tendon, and similarly the groove between the fibula and the tendon on the lateral aspect of the ankle. Inflammation of an adventitious subcutaneous bursa is easily visible as a tender localised swelling over the posterior surface of the os calcis, but a bursitis of the calcaneal bursa is less obvious, being seen only as a small localised swelling just above the insertion of the tendon.

\section{Aetological Considerations.}

\section{The Major Lesion}

The most important predisposing factor is increasing age; complete lesions are comparitively uncommon in young athletes even in conditions of great stress. Stress is obviously important, but very great strains can be tolerated without ill effect provided they are applied slowly. If the load is applied abruptly and particularly if it takes the subject by surprise, so that the muscle is actively contracting at the moment when a great stretching force is applied, then rupture is much more apt to occur. However, in these circumstances in the younger athlete particularly, failure is more likely either in the muscle belly or more commonly at the musculo-tendinous junction. Gastrocnemius is usually affected as it has the disadvantage of working across two joints, a situation which confuses a muscle and commonly predisposes to tearing e.g. hamstrings, rectus femoris and biceps.

The role of cortico-steroids is not clearly defined. As noted above Ljungquist could not find evidence that steroid injections were responsible in more than one of his cases, but other suggestive case reports exist. There is clearly a need for more experimental evidence before the strong views expressed by Sweetnam (1969) can be justified but it is very likely that weakening of the tendon fibres due to suppression of collagen formation and interruption of blood flow are important factors.

\section{The Minor Lesion.}

Minor tendon lesions may originate from sudden severe strain or it could be that they result from repeated strain producing a fatigue situation. Thus as Williams has noted (1967), lesions are common when an athlete changes from cross country running to track running.

In lesions of the paratenon and surrounding structures and in the minor tendon lesions, other factors operate.

The important factors are probably:-

1. Th e change from heel to toe foot strike as in cross country running, to toe strike as in short distance track events. Two separate thrusts are transmitted through the tendon at each stride, the forced dorsiflexion on alighting and the smoother plantar flexion on stepping off.

2. Change in footwear may be equally important. Firstly cross country and road running shoes commonly have a heel whereas track shoes have no heel at all, so that the ankle is in greater dorsi-flexion, and the Achilles? tendon under greater tension. Secondly there is little padding in a track shoe io cushion the contact with the ground.

Another important factor is the hardness of the running surface. Very hard surfaces are apt to precipitate symptoms, perhaps because lack of resilience necessitates much greater elastic recoil within the triceps surae muscle and its tendon, i.e. soleus and the two heads of gastrocnemius.

Finally ill-fitting shoes or boots may cause a continual friction on the heel, leading ultimately to either the formation of a sub-cutaneous bursa or to bursitis in the bursa lying between the tendon and the os calcis. Tight lacing of boots around the ankle is particularly liable to cause the latter.

\section{References}

1. Elliott, D. H. (1965) “Structure and Function of Mammalian Tendon”. Biol. Rev. 40, 392

2. Goldman, S. Linscheid, R. L. \& Bickel W. H. (1969) “Disruption of the Tendo Achilles”. Mayo Clin. Proc. 44, 28 
3. Ljungquist, R. (1968) "Subcutaneous Partial Rupture of theAchilles Tendon". Acta Orth. Scand. Suppl. 113, 23

4. Arner, O. Lindholm, A. \& Orell, S. R. (1958/9) "Histological Changes in Subcutaneous Rupture of the Achilles Tendon". Acta. Chir. Scand. 116, 484

5. Ljungquist, R. (1968) "Subcutaneous Partial Rupture of the Achilles Tendon". Acta Orth. Scand. Suppl. 113,18

6. Sweetnam, R. “Corticosteroid Arthropathy and Tendon Rupture”. Editorial (1969) J.B.J.S. 51B 3, 397

7. Williams, J. G. P. (1967) "Cause and Prevention of the Achilles Peritendonitis in Middle and Long Distance Runners". B.J. Sports Med. 3, 16

\section{BOOK REVIEW}

Physiology for Students and Teachers of Physical Education

A. W. SLOAN, B.Sc., M.D., Ph.D., F.R.C.P.G., M.R.C.P., F.R.S.S.Afr.

Professor of Physiology, University of Cape Town.

Formerly Senior Lecturer in Physiology, University of Glasgow.

Edward Arnold Ltd., London, 1970. £3.

According to the preface, the purpose of this book is to present human physiology in simple terms emphasizing those factors essential to the maintenance of health and the physiological effects of exercise.

The first three chapters are introductory, being concerned with cellular physiology and basic but essential biochemistry and biophysics. These opening chapters are presented so that the student with an elementary science background will find little difficulty in comprehending the information. The succeeding chapters are concerned with the physiology of the body systems and the information is well set out with clear subheadings. The knowledge within each chapter is pertinent and expressed succinctly. The effects of exercise are noted in relation to each system and are summarized in the chapters on Exercise and Training.

Perhaps the main improvement could be in the realistic use of colour in the diagrams and illustrations which appear only in black and white. Another shortcoming is the lack of documentation in the chapters on Exercise; Training; and Life in Extreme Environments. However, there is a useful list of recommended readings at the end of the book. In one chapter the author deals with tests of physical fitness but fails to discuss the physiological significance or validity of these tests. In addition, the reliability and objectivity of many of the tests suggested are questionable. It is also unfortunate that the misuse of the term "isotonic contraction", in relation to the shortening of a muscle, is perpetuated.

As a text of basic physiology and an introduction to exercise physiology, which is designed as a companion volume to "Anatomy for Students and Teachers of Physical Education", by J. W. Perrott, this book will serve its purpose in meeting the needs of physical education students and teachers.

Paul D. Robinson, B.Sc., D.L.C., M.A., Ph.D. 